\title{
Current Status and Development Trend of Nanoscale Generator
}

\author{
Fuzhong Zheng
}

\author{
Sichuan University of Science \& Engineering, College of Mechanical Engineering, Sichuan \\ 643000, P. R. China. \\ zfz@suse.edu.cn
}

\begin{abstract}
. nano generator is a kind of generator based on nanometer magnitude, which can transform certain form of mechanical energy into electrical energy. In this paper, the current research status of nanoscale generator is reviewed, the classification and working principle of nanoscale generator are explained, and several representative nanoscale generators are introduced. At the end of this paper, some problems of nanoscale generator are analyzed, and the development trend of nanoscale generator is predicted. It is considered that nanoscale generator will be focused on optimizing the power generation material, advancing the power generation process and improving the performance of the equipment.
\end{abstract}

Keywords: nano-generator, status quo, development trend.

\section{Classification and Working Principle of Nanoscale Generator}

\subsection{Piezoelectric Nanoscale Generator}

Piezoelectric nanometers convert the mechanical energy of compression and bending into electrical energy. In the process of generating electricity, Zinc Oxide is used as a special nano material. The special technology of Zinc Oxide is used to prepare nanomaterials, which will have dual effects of semiconductor and piezoelectric. The piezoelectric effect of Zinc Oxide makes it able to output unidirectional current, because when Zinc Oxide is in contact with metal, the electron escape work function of the platinum electrode is greater than that of Zinc Oxide, and the electron will flow from the Zinc Oxide electrode to the platinum electrode; the outside of the line nanowire forms an electric field from the platinum electrode to the Zinc Oxide direction, and the electronic flow inside the nanowire. And then the current is formed. At present, the research shows that the low density Zinc Oxide line produces direct current, and the vertical Zinc Oxide nanowire with high density generates alternating current. For this reason, the density and space condition (tilt or vertical) of Zinc Oxide nanowire can be determined according to actual needs. The current waveforms generated by piezoelectric nanometers are shown below.

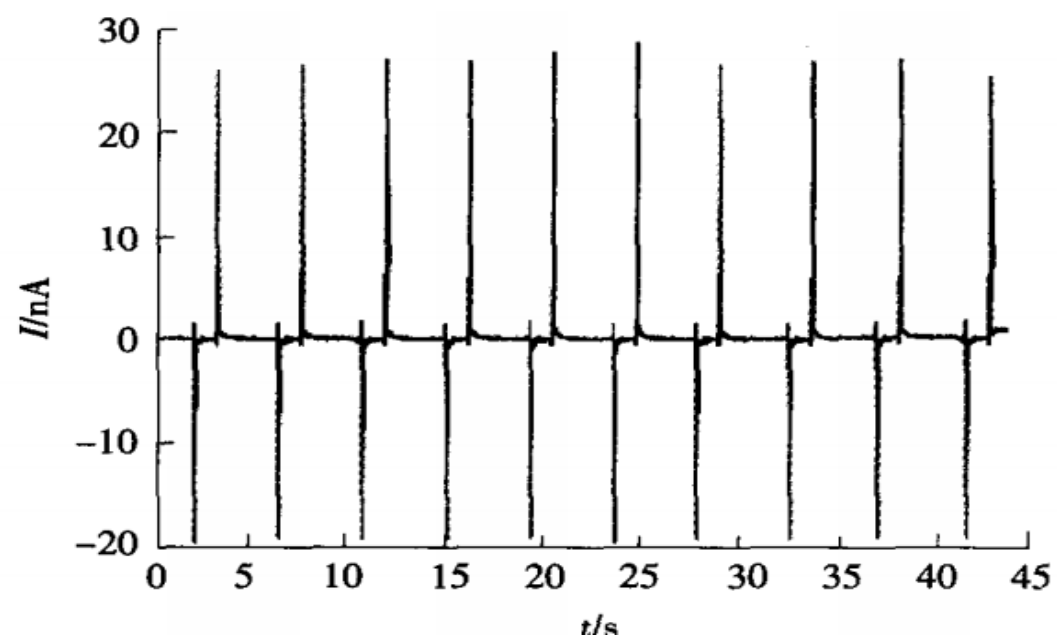

Figure. 1 current waveform of a piezoelectric nanoscale 


\subsection{Friction Nanoscale Generator}

Nanomaterials used in friction nanogenerators are nylon and polytetrafluoroethylene. When nylon is in contact with polytetrafluoroethylene and has some friction, the one end of the polytetrafluoroethylene will get electrons., As the two surfaces in a non-contact state needs to be electrically neutral, The electrons will flow from one end of polytetrafluoroethylene to the nylon end and then form a current while a slip between the two materials,; when the two materials are in contact again, The contact surfaces should still be electrically neutral, which requires the electrons flowing back from the outflow to keep the electricity neutral. Thus, the current will be formed inside and outside the circuit. The friction nanoscale is another breakthrough technology developed by Professor Wang Zhonglin's team. For the single layer friction nanoscale of $3 \mathrm{~cm} 2$, the output voltage can reach 200-1000V, and the high voltage can load hundreds of LED lamps and sensors. In addition, Professor Zhang Haixia, of the school of information science and technology of Peking University, led his team to apply "flexible printed circuit technology" to the production of friction nanoscale generators, which has achieved mass production of friction nanoscale generators. The relationship between fabrication and application of the friction nano-generator is shown as follows:

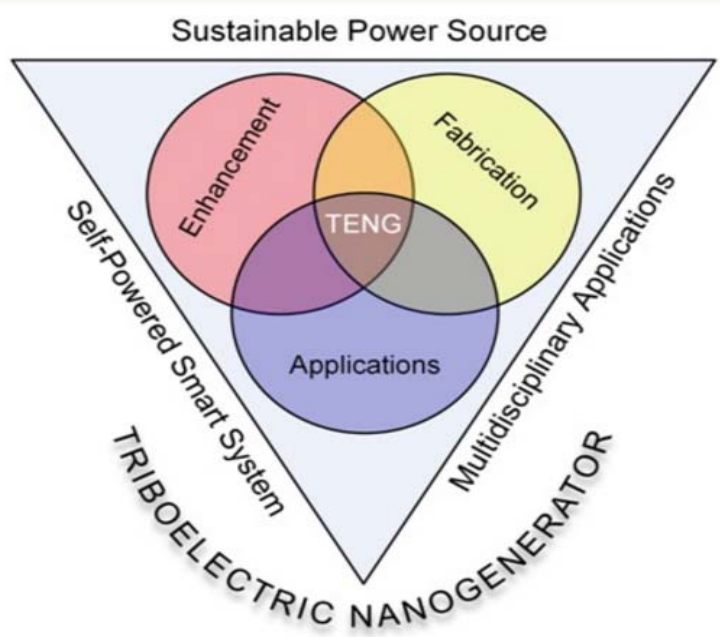

Figure. 2 The relationship between fabrication and application of the friction nano-generator

\subsection{Pyroelectric Nanoscale Generator}

Pyroelectric nanoscale is a kind of energy collection device, which uses nanostructured pyroelectric materials (usually with nanostructured ferroelectric materials, such as lead zirconate titanate, Zinc Oxide, etc.) to convert external heat energy into electrical energy. The working principle of pyroelectric nanometers can be explained in two cases: the first pyroelectric effect and the second pyroelectric effect. The first pyroelectric effect explains the charge produced in the absence of strain, and it exists in a series of ferroelectric materials (PZT, BTO). The electric dipole with thermal induction is randomly moving (oscillating) on its own balance axis, and the electric dipole increases with the increase of ambient temperature. When the ambient temperature changes, the spontaneous polarization of the ferroelectric material will change, and the external circuit will generate current. Generally, the voltage produced by the pyroelectric nanoscale is larger, but its output current is small, so in practice the pyroelectric nanoscale is mostly used as a potential power source. In addition, pyroelectric nanometers are also used as active sensors to measure ambient temperature. The Second pyroelectric effect explains the existence of strain charges due to thermal expansion, which exists in some wurtzite structural materials. Thermal expansion will cause piezoelectric potential difference in ferroelectric materials, which will drive the external circuit to generate electric current. 


\section{Comparison of the Properties of the Three Kinds of Nanoscale Generators}

This article mainly introduces the power generation characteristics of the three kinds of nano generators. The three kinds of nanometers have uniform properties, namely, high output voltage and low output current. At the same time, the nanometers use the characteristics of the semiconductor to generate electricity, and the internal resistance is relatively large, which is a main reason for their relatively small output current. Compared to the pyroelectric nanoscale and the piezoelectric nanoscale, the current output of the friction nanoscale generator is larger, and it is the most potential nanoscale generator at present. The characteristic parameters of the three generators are presented as follows:

Table. 1 Comparison of Generation Characteristics of Three Nanogenerators

\begin{tabular}{cccc}
\hline type & Size $\left(\mathrm{cm}^{2}\right)$ & Open circuit voltage $(\mathrm{V})$ & current density $\left(\mu \mathrm{A} \cdot \mathrm{cm}^{-2}\right)$ \\
\hline \multirow{3}{*}{ Piezoelectricity } & 12.25 & 200 & 150 \\
& 0.5 & 9.8 & 1.4 \\
& 1 & 8 & 0.8 \\
Tribological type & 314 & 850 & 9.55 \\
& 8 & 465 & 13.4 \\
& 58.06 & 1200 & 3.4 .45 \\
Pyroelectric & 2.52 & 22 & 30.171 \\
& 0.62 & 2.5 & 0.57 \\
\hline
\end{tabular}

\section{Development Trend Analysis of Nanoscale Generator}

In view of the most common three kinds of nanoscale generators at the present stage, their power generation principles are different, which results in their different characteristics of the power generation function, and different nanoscale generators have different adaption environments. Piezoelectric nano generators are generally used in the environment with vibration conditions, and the installation process is more complex, which has certain limitations. Friction nanogenerators generate higher efficiency, but they need to use specific materials for friction to generate electricity. Pyroelectric generators are required to be used in environments with high temperature differences, but the energy generated by them is relatively low and their development potential is relatively small. From the invention of nanodynamo to its application, the technology of nano generator has made great progress (it can achieve the conversion rate of 17-30\%). It is predicted that nanoscale generators will be improved and perfected in the fields of power generation materials, power generation, circuit optimization design and composite nanoscale generator in the future, when the utility of nanoscale generator will be further enhanced.

\section{Summary}

To sum up, this paper mainly introduces the working principles of piezoelectric nanoscale, friction nanoscale and pyroelectric nanoscale. After comparing and analyzing the generation characteristics of the three kinds of generators, we are clear about their different properties and adaption environment. Finally, the development trend of nanoscale generator is predicted that the technology of nanoscale generator will be improved and developed in the direction of power generation materials, power generation technology and circuit optimization.

\section{References}

[1]. Chen Zhimin, Rong Xun, Cao Guangzhong. Research status and development trend of nanomaterials [J]. micro nano electronic technology, 2016, 53 (1): 36-42.

[2]. Guo Yin, Zhang Qinghong, Li Yaogang, et al. Research progress of wearable friction nanodynamo [J]. [J]., China, 2016, 35 (2): 
[3]. Wang Zhong Lin. Principles and potential applications of piezoelectric nanogenerators. [J]. physics, 2006, 35 (11): 897-903.

[4]. Zhang Yan, Wang Zhonglin, Hu you fan, et al. Nano generator, nano generator set and its self power supply system: CN $102683573 \mathrm{~A}[\mathrm{P}] .2012$. 